\title{
Markers of pediatric respiratory distress predictive of poor outcome in low- and middle-income countries: a systematic review
}

\author{
Anna B Hedstrom ${ }^{1}$ (D), Amelie O von Saint Andre-von Arnim ${ }^{1}$ (D), Kalee L Grassia ${ }^{2}$, Katie R Nielsen ${ }^{3}$ (D) \\ ${ }^{1}$ University of Washington Department of Pediatrics, Seattle, Washington USA; Seattle Children's Hospital, Seattle, Washington USA; University of \\ Washington Department of Global Health, Seattle, Washington USA, 2 Seattle Children's Hospital, Seattle, Washington USA, 3 Seattle Children's \\ Hospital, Seattle, Washington; University of Washington Department of Global Health, Seattle, Washington USA USA; \\ Keywords: child mortality, triage, pneumonia, pediatric \\ https://doi.org/10.29392/001c.14136
}

\section{Journal of Global Health Reports}

Vol. 4, 2020

\section{Background}

Lower respiratory tract diseases remain significant causes of pediatric mortality in lowand middle-income countries. In these settings, staff must quickly triage patients for timely initiation of treatment and potential transfer to higher levels of care. World Health Organization (WHO) guidelines focus on two physical exam findings - tachypnea and chest indrawing - without addressing the multitude of other respiratory assessment tools used by healthcare workers. This lack of additional validated markers makes triage of patients challenging. The aim of this study was to systematically review respiratory assessments in children under five years of age that have been associated with poor clinical outcomes in resource limited settings.

\section{Methods}

We conducted a systematic search for studies published between January 1, 2008 and January 21, 2018 using Ovid MEDLINE and Embase including patients five years of age and younger. Major categories of search terms were "respiratory distress", "respiratory symptoms", "low- and middle-income countries" and "clinical assessment". We extracted data relevant to study characteristics, respiratory assessments and clinical outcomes.

\section{Findings}

Out of 2317 identified publications, 63 full text articles fit inclusion criteria, 56 reported statistically significant associations and were included in analyses and these publications included 53 unique study populations. Publications were from: low-income countries (26\%), lower middle-income countries (55\%) and upper middle-income countries (19\%). The most common respiratory assessments were hypoxia (50\%), tachypnea (46\%) and chest indrawing/retractions (38\%). Death was the most frequently reported clinical outcome (54\%), followed by hypoxia (23\%). Hypoxia, chest indrawing/retractions and tachypnea were the most commonly reported risk factors for mortality.

\section{Conclusions}

Hypoxia, chest retractions and tachypnea are key risk factors for mortality form pediatric respiratory disease in resource limited settings. Death is the primary outcome of interest for research in this area. As pediatric respiratory interventions are deployed in these settings, better understanding of which respiratory assessments are predictive of poor clinical outcomes could guide management and transfer of care decisions.

Lower respiratory tract diseases remain significant causes of pediatric mortality globally, accounting for $16 \%$ of deaths under age five. ${ }^{1-3}$ The burden of respiratory mortality is greatest in low- and middle-income countries (LMICs) where many health facilities lack sufficient pediatric acute care resources. ${ }^{4,5}$ Staff in resource-limited facilities must quickly triage patients using available assessment techniques for timely initiation of treatment and potential transfer to higher levels of care. World Health Organization
(WHO) guidelines for care of sick children focus on two physical exam findings- tachypnea and chest indrawingwithout addressing the multitude of other respiratory assessment tools used by healthcare workers. ${ }^{6,7}$ The lack of additional validated markers of respiratory distress linked to relevant clinical outcomes makes triage of these pediatric patients challenging. ${ }^{8}$

Treatment options for pediatric respiratory distress including oxygen, continuous positive airway pressure (CPAP) 
and high flow nasal canula (HFNC) are increasingly available in LMICs; however, evidence-based treatment algorithms for initiation and use of these therapies are lacking. ${ }^{9,10}$ Several studies have looked at the correlation of respiratory symptoms or composite scores to important single clinical markers which may indicate therapy is needed. A meta analysis of children with lower respiratory tract infections, however, did not find a single clinical sign or symptom that accurately and reliably identified hypoxemia. ${ }^{11} \mathrm{Al}-$ though scores of dyspnea are in use to guide management of asthma and bronchiolitis, they have undergone limited and inadequate validation for disease severity. ${ }^{12,13}$ Additionally, many resource-limited settings (RLS) lack technology to perform advanced diagnostics used to guide therapy, such as blood gas analysis and pulmonary function tests. As pediatric respiratory interventions are deployed in RLS, understanding which respiratory assessments are predictive of poor clinical outcomes could guide management and transfer of care decisions.

The objective of this review is to determine which respiratory assessments in children under five years of age have been associated with poor clinical outcomes in RLS.

\section{METHODS}

We conducted a systematic review of the literature following the PRISMA checklist. ${ }^{14}$ We conducted a search for studies published between January 1, 2008 and January 21, 2018 using Ovid MEDLINE (Online Supplementary Document, Appendix S1) and Embase.com. All results were limited to English, German or Spanish languages and ages 0-5. Major categories of search terms were "respiratory distress", "respiratory symptoms", "low- and middle-income countries (LMIC)" and "clinical assessment”.

\section{ELIGIBILITY CRITERIA}

Publications that met the following criteria were included:

1. Took place in a low- or middle-income country as defined by the World Bank, July 2019. ${ }^{15}$

2 . $\geqslant 50 \%$ of subjects were under five years of age.

3. Evaluation of subjects occurred in a hospital, emergency department or outpatient clinic setting (not in a home).

4. Respiratory diagnostic assessment, including physical exam, vital sign, laboratory test and/or diagnostic/imaging study, was performed (no parent report symptoms) .

5. Association between a respiratory diagnostic assessment and a poor clinical outcome (including death, clinical course, transfer, monitoring or laboratory result and/or treatment requirement) was reported.

Publications that met the following criteria were excluded:

1. Not original research (comments, editorials, letters and/or notes).

2. Systematic reviews.

3. Case reports or case series with $<30$ patients.

4. Respiratory diagnostic assessment involved advanced equipment, such as respiratory viral PCR and/or pulmonary function testing.

5. Clinical outcome was a diagnosis, such as pneumonia and/or bronchiolitis.

\section{DATA EXTRACTION}

Citations were managed with Covidence (Copyright (c) 2019 The Cochrane Collaboration). ${ }^{16}$ Five reviewers screened and discussed 100 abstracts to reach consensus on inclusion and exclusion criteria. Four reviewers screened an additional 30 abstracts, of which $60 \%$ were included/rejected unanimously with an inter-rater reliability of 0.57 . Remaining abstracts were screened by one reviewer, and included abstracts underwent full text review. Two reviewers reviewed and reached consensus on inclusion of full text publications and categorization of bias. Disagreement on study inclusion/ exclusion, data selection and bias assessment were resolved by discussion between the two reviewers. If no concensus was found between two reviewers, discussions between all review authors was used to resolve the disagreement. Data was extracted into REDCap (8.11.9- (C2019 Vanderbilt University). ${ }^{17}$

\section{ASSESSMENT OF BIAS}

We used the Risk of Bias Assessment tool for Non-randomized Studies (RoBANS) score to objectively evaluate bias in six domains, ${ }^{18}$ namely:

1. Selection of participants - Selection biases caused the inadequate selection of participants

2. Confounding variables - Selection biases caused by the inadequate confirmation and consideration of confounding variables.

3. Measurement of exposure - Performance biases caused by inadequate measurements of exposure.

4. Blinding of outcome assessments - Detection biases caused by the inadequate blinding of outcome assessments.

5. Incomplete outcome data - Attrition biases caused by the inadequate handling of incomplete outcome data.

6. Selective outcome reporting - Reporting biases caused by the selective reporting of outcomes.

For each domain, a study was determined to have "low risk," "high risk," or "unclear risk" of bias according to specific score criteria. Two reviewers performed bias assessment on each publication.

\section{DATA SYNTHESIS}

Given the heterogeneity of publications, a meta-analysis was not performed. In line with the review objective, we focused analysis on publications reporting a statistically significant association between an assessment and outcome for reporting results. The data were summarized descriptively, stratified by country income level. ${ }^{15}$ When $>1$ publication described identical study populations (study location, age group, inclusion criteria, study dates), the study population was only included once in Table 2 (summary characteristics). All other analyses report data from each in- 


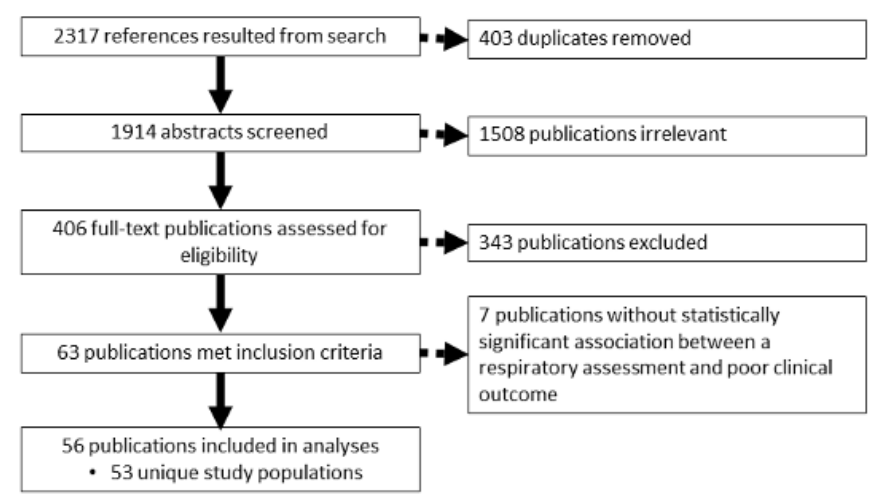

Figure 1: Flowchart of Study Evaluation

dividual publication regardless of population overlap. "Statistical significance" was determined by the authors of the manuscripts.

\section{RESULTS}

Of 2317 identified publications, 1914 abstracts were screened, and 406 underwent full text review. 343 publications were excluded after full text review because: they did not fit inclusion criteria (79\%), no full texts were available (12\%) or the content was not relevant (9\%). 56 of 63 full text publications reported statistically significant associations and were included in analyses (Figure 1) - representing 53 unique study populations. Kuti ${ }^{19-21}$ and Chisti ${ }^{22,23}$ published multiple manuscripts using identical study populations. Two additional publications by Chisti described study populations enrolled during almost identical study periods at the same site and some patients may have been included in both publications. ${ }^{24,25}$ We did not receive author confirmation about this question, so these populations were considered unique in this analysis. Relevant findings of each publication, stratified by country income level, are summarized in Table 1 (plates A, B and C) with more details found in the Online Supplementary Document, Appendix S2. 
Table 1A. Low income country publications included

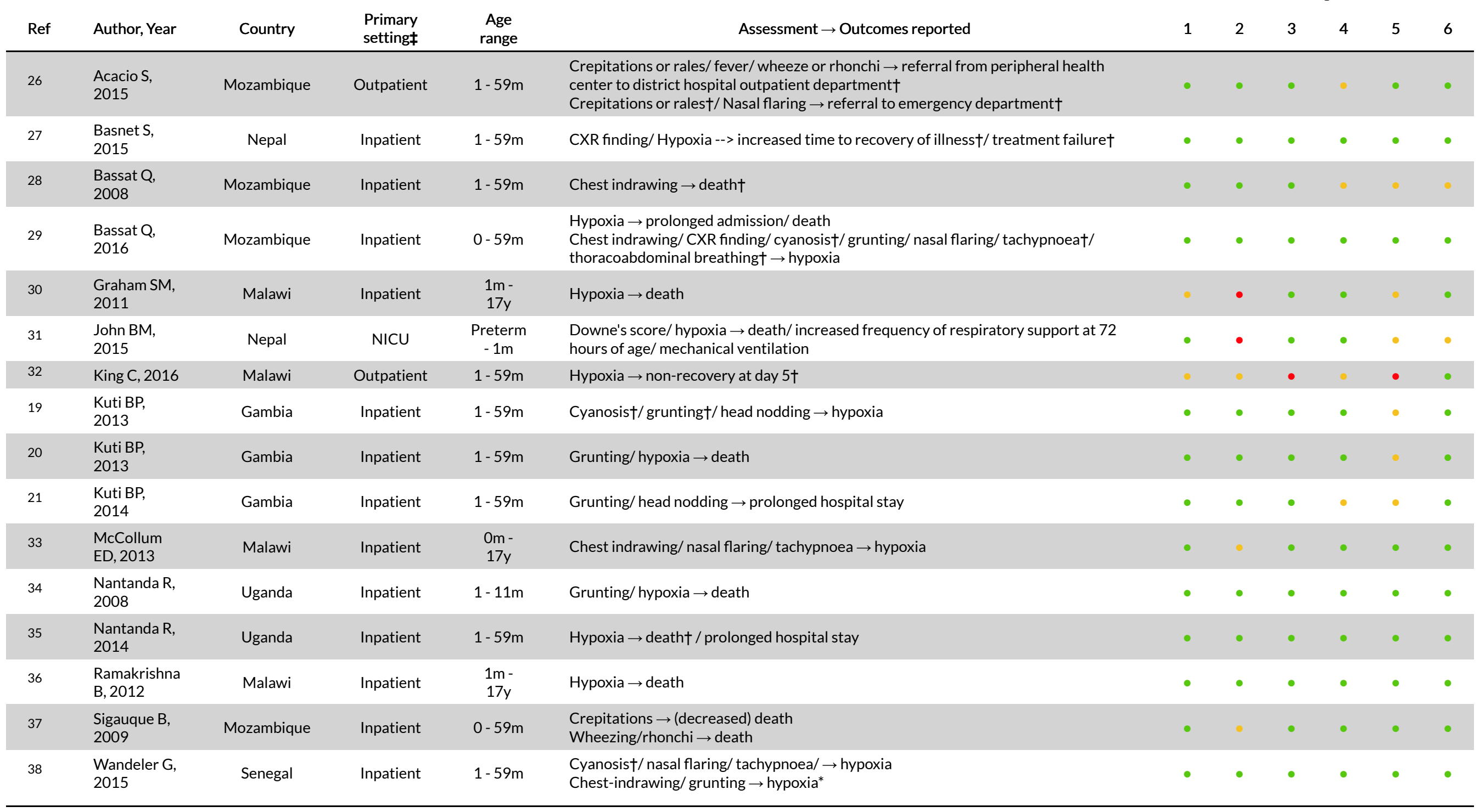

$=$ low risk of bias, $\odot=$ unclear risk of bias, $\bullet=$ high risk of bias

$\uparrow$ Association significant on multivariate analysis. ₹ Primary Setting. Inpatient = Inpatient general ward. PICU = Pediatric Intensive Care Unit. Emergency = Emergency Department. Outpatient $=$ Outpatient clinic

$\S$ Bias categories: 1.) Comparibility of participants 2.) Confounding variables 3.) Measurement of Exposure 4.) Blinding of outcome assessments 5.) Incomplete outcome data 6.) Selective outcome reporting 
Table 1B. Lower middle income country publications included

\begin{tabular}{|c|c|c|c|c|c|c|c|c|c|c|c|}
\hline \multirow[b]{2}{*}{ Ref } & \multirow[b]{2}{*}{ Author, Year } & \multirow[b]{2}{*}{ Country } & \multirow[b]{2}{*}{$\begin{array}{l}\text { Primary } \\
\text { setting }\end{array}$} & \multirow[b]{2}{*}{$\begin{array}{c}\text { Age } \\
\text { range }\end{array}$} & \multirow[b]{2}{*}{ Assessment $\rightarrow$ Outcomes reported } & \multicolumn{6}{|c|}{ Bias categories§ } \\
\hline & & & & & & 1 & 2 & 3 & 4 & 5 & 6 \\
\hline 39 & $\begin{array}{l}\text { Abdulkadir } \\
\text { MB, } 2015\end{array}$ & Nigeria & Inpatient & $1-59 m$ & Hypoxia $\rightarrow$ death/ prolonged hospitalization & - & - & $\bullet$ & - & $\bullet$ & $\bullet$ \\
\hline 40 & $\begin{array}{l}\text { Addo-Yobo } \\
\mathrm{E}, 2011\end{array}$ & $\begin{array}{l}\text { Bangladesh } \\
\quad+3\end{array}$ & Outpatient & $1-59 m$ & Tachypnoea $\rightarrow$ treatment failure $\dagger$ & - & $\bullet$ & $\bullet$ & $\bullet$ & $\bullet$ & $\bullet$ \\
\hline 41 & $\begin{array}{l}\text { Agweyu A, } \\
2014\end{array}$ & Kenya & Inpatient & $1-59 m$ & Grunting $\rightarrow$ treatment failure & - & - & $\bullet$ & - & 。 & $\bullet$ \\
\hline 42 & $\begin{array}{l}\text { Agweyu A, } \\
2018\end{array}$ & Kenya & Inpatient & $1-59 m$ & Fever/lower chest wall indrawing/tachypnoea $\rightarrow$ death $\dagger$ & - & $\bullet$ & - & $\bullet$ & $\bullet$ & $\bullet$ \\
\hline 43 & $\begin{array}{l}\text { Asghar R, } \\
2008\end{array}$ & $\begin{array}{l}\text { Bangladesh } \\
+6\end{array}$ & Inpatient & $1-59 m$ & Hypoxia $\rightarrow$ death/ treatment failure $\dagger$ & - & $\bullet$ & $\bullet$ & - & - & $\bullet$ \\
\hline 44 & $\begin{array}{l}\text { Ashraf H, } \\
2012\end{array}$ & Bangladesh & Outpatient & $1-59 m$ & Hypoxia/ tachypnoea $\rightarrow$ clinical failure & $\bullet$ & $\bullet$ & - & $\bullet$ & - & $\bullet$ \\
\hline 45 & $\begin{array}{l}\text { Awasthi S, } \\
2008\end{array}$ & India & Outpatient & $1-59 m$ & Tachypnoea $\rightarrow$ clinical failure $\dagger$ & - & $\bullet$ & $\bullet$ & $\bullet$ & - & $\bullet$ \\
\hline 46 & $\begin{array}{l}\text { Benet T, } \\
2017\end{array}$ & India + 3 & Inpatient & $1-59 m$ & $\begin{array}{l}\text { CXR finding/ cyanosis/ lower chest indrawing/ (lack of) rasping } \rightarrow \text { hypoxia } \\
\text { Hypoxia } \rightarrow \text { death/ prolonged hospitalization }\end{array}$ & $\bullet$ & $\bullet$ & $\bullet$ & $\bullet$ & $\bullet$ & $\bullet$ \\
\hline 47 & $\begin{array}{l}\text { Bharti B, } \\
2008\end{array}$ & India & Inpatient & $\begin{array}{l}1 \mathrm{~m}- \\
17 y\end{array}$ & CXR finding $\rightarrow$ length of stay & - & - & - & $\bullet$ & - & $\bullet$ \\
\hline 23 & $\begin{array}{l}\text { Chisti MJ, } \\
2011\end{array}$ & Bangladesh & Inpatient & $1-59 m$ & Hypoxia $\rightarrow$ death $\dagger$ & - & $\bullet$ & $\bullet$ & $\bullet$ & $\bullet$ & $\bullet$ \\
\hline 22 & $\begin{array}{l}\text { Chisti MJ, } \\
2012\end{array}$ & Bangladesh & Inpatient & $1-59 m$ & Lower chest indrawing/ nasal flaring $\rightarrow$ hypoxiat & - & - & - & $\bullet$ & $\bullet$ & $\bullet$ \\
\hline 24 & $\begin{array}{l}\text { Chisti MJ, } \\
2013\end{array}$ & Bangladesh & $\mathrm{PICU}$ & $0-59 m$ & Hypoxia $\rightarrow$ death $\dagger$ & $\bullet$ & $\bullet$ & $\bullet$ & $\bullet$ & $\bullet$ & $\bullet$ \\
\hline 25 & $\begin{array}{l}\text { Chisti MJ, } \\
2013\end{array}$ & Bangladesh & Inpatient & $0-59 m$ & Lower chest wall indrawing $\dagger /$ tachypnoea $\rightarrow$ hypoxia & - & $\bullet$ & - & $\bullet$ & $\bullet$ & • \\
\hline 48 & $\begin{array}{l}\text { Chudasama } \\
\text { RK, } 2012\end{array}$ & India & Inpatient & $1-59 m$ & Fever $\rightarrow$ death/intensive care unit admission & $\bullet$ & $\bullet$ & - & $\bullet$ & $\bullet$ & $\bullet$ \\
\hline 49 & $\begin{array}{l}\text { El Kholy AA, } \\
2014\end{array}$ & Egypt & Inpatient & $\begin{array}{l}1 \mathrm{~m}- \\
17 y\end{array}$ & Cyanosis $\rightarrow$ prolonged length of hospital stay & - & - & - & $\bullet$ & 。 & $\bullet$ \\
\hline 50 & $\begin{array}{l}\text { Emukule } \\
\text { GO, } 2014\end{array}$ & Kenya & Inpatient & $0-59 m$ & $\begin{array}{l}\text { Tachypnoea } \rightarrow \text { (decreased) death } \\
\text { Chest wall indrawing } \dagger / \text { hypoxia/ nasal flaring/ wheezing } \rightarrow \text { death }\end{array}$ & $\bullet$ & $\bullet$ & $\bullet$ & - & $\bullet$ & $\bullet$ \\
\hline 51 & $\begin{array}{l}\text { Gowraiah V, } \\
2014\end{array}$ & India & Inpatient & $0-59 m$ & Tachypnoea $\rightarrow$ death & - & $\bullet$ & - & 。 & 。 & 。 \\
\hline 53 & $\begin{array}{l}\text { Ibraheem } \\
\text { RM, } 2014\end{array}$ & Nigeria & Inpatient & $1-59 m$ & $\begin{array}{l}\text { Hypoxia } \rightarrow \text { difficult breathing } \\
\text { Abnormal percussion note/ bronchial breath sounds/cyanosis/grunting/intercostal } \\
\text { retractions/lower chest indrawing } \rightarrow \rightarrow \text { hypoxia }\end{array}$ & - & - & - & - & 。 & $\bullet$ \\
\hline 54 & $\begin{array}{l}\text { Jroundi I, } \\
2014\end{array}$ & Morocco & Inpatient & $1-59 m$ & $\begin{array}{l}\text { CXR finding/ cyanosis } \dagger \text { / fever/ hypoxia/ tachypnoea/ wheezing } \rightarrow \text { poor prognosis } \\
\text { (death, intensive care or Respiratory Index Severity Children (RISC) score } \geq 3 \text { ) }\end{array}$ & $\bullet$ & $\bullet$ & - & - & - & • \\
\hline 55 & Mahajan V, & India & Emergency & 1-59m & Chest retractions/ fever/grunting/ hypoxia/ tachypnoea $\rightarrow$ hospital admission $\dagger$ & $\bullet$ & $\bullet$ & $\bullet$ & - & $\bullet$ & $\bullet$ \\
\hline
\end{tabular}




\begin{tabular}{|c|c|c|c|c|c|c|c|c|c|c|c|}
\hline & 2016 & & & & & & & & & & \\
\hline 56 & $\begin{array}{l}\text { Morgan MC, } \\
2017\end{array}$ & Kenya & $\mathrm{NICU}$ & $\begin{array}{l}\text { Preterm } \\
-28 \mathrm{~d}\end{array}$ & $\begin{array}{l}\text { Grunting/ nasal flaring/retractions } \rightarrow \text { hypoxiat } \\
\text { Hypoxia/ nasal flaring } \rightarrow \text { death } \dagger\end{array}$ & $\cdot$ & $\bullet$ & - & $\bullet$ & $\bullet$ & $\bullet$ \\
\hline 57 & $\begin{array}{l}\text { Mwaniki } \\
\text { MK, } 2009\end{array}$ & Kenya & Inpatient & $0-59 m$ & Hypoxia $\rightarrow$ death $\dagger$ & $\bullet$ & $\bullet$ & $\bullet$ & - & $\bullet$ & - \\
\hline 58 & $\begin{array}{l}\text { Naheed A, } \\
2009\end{array}$ & Bangladesh & Inpatient & $0-59 m$ & $\begin{array}{l}\text { Tachypnoea } \rightarrow \text { (decreased) death } \\
\text { Chest indrawing } \rightarrow \text { death }\end{array}$ & $\bullet$ & - & - & $\bullet$ & $\bullet$ & - \\
\hline 59 & $\begin{array}{l}\text { Orimadegun } \\
\mathrm{AE}, 2013\end{array}$ & Nigeria & Inpatient & $0-17 y$ & Chest retractions/ fast or difficult breathing/grunting/ nasal flaring $\rightarrow$ hypoxia & $\bullet$ & - & - & - & - & - \\
\hline 60 & $\begin{array}{l}\text { Quiambao } \\
\text { BP, 2009 }\end{array}$ & Philippines & Inpatient & $0-11 \mathrm{~m}$ & Apnea/ CXR finding/ chest indrawing $\rightarrow$ death & $\bullet$ & - & $\bullet$ & $\bullet$ & $\bullet$ & $\bullet$ \\
\hline 61 & $\begin{array}{l}\text { Rao YK, } \\
2012\end{array}$ & India & Emergency & $1-59 m$ & Cyanosis/grunting/ nasal flaring/ tachypnoea $\rightarrow$ hypoxia & - & - & - & - & - & - \\
\hline 62 & $\begin{array}{l}\text { Singla G, } \\
2015\end{array}$ & India & $\mathrm{NICU}$ & $\begin{array}{l}\text { Preterm } \\
-1 \mathrm{~m}\end{array}$ & $\begin{array}{l}\text { Low respiratory rate/ hypoxia } \rightarrow \text { death } \\
\text { Abnormal respiratory rate/ hypoxia/ chest recessions/ respiratory distress } \rightarrow \text { prolonged } \\
\text { hospitalization } \\
\text { Cyanosis } \rightarrow \text { shortened hospitalization in surviving neonates }\end{array}$ & - & - & - & - & - & - \\
\hline 63 & $\begin{array}{l}\text { Webb C, } \\
2012\end{array}$ & Kenya & Inpatient & $1-59 m$ & Hypoxia $\rightarrow$ treatment failure & - & • & - & - & $\cdot$ & $\bullet$ \\
\hline 64 & $\begin{array}{l}\text { Wilson PT, } \\
2017\end{array}$ & Ghana & Inpatient & $1-59 m$ & Hypoxia/ tachypnoea $\rightarrow$ death $\dagger$ & $\bullet$ & $\bullet$ & $\bullet$ & - & $\bullet$ & $\bullet$ \\
\hline
\end{tabular}

$\bullet=$ low risk of bias, $\oslash$ = unclear risk of bias, $\bullet=$ high risk of bias

${ }^{\dagger}$ Association significant on multivariate analysis. ₹ Primary Setting: Inpatient = Inpatient general ward. PICU = Pediatric Intensive Care Unit. Emergency = Emergency Department. Outpatient = Outpatient clinic $\S$ Bias categories: 1.) Comparibility of participants 2.) Confounding variables 3.) Measurement of Exposure 4.) Blinding of outcome assessments 5.) Incomplete outcome data 6.) Selective outcome reporting 
Table 1C. Upper middle income country publications included

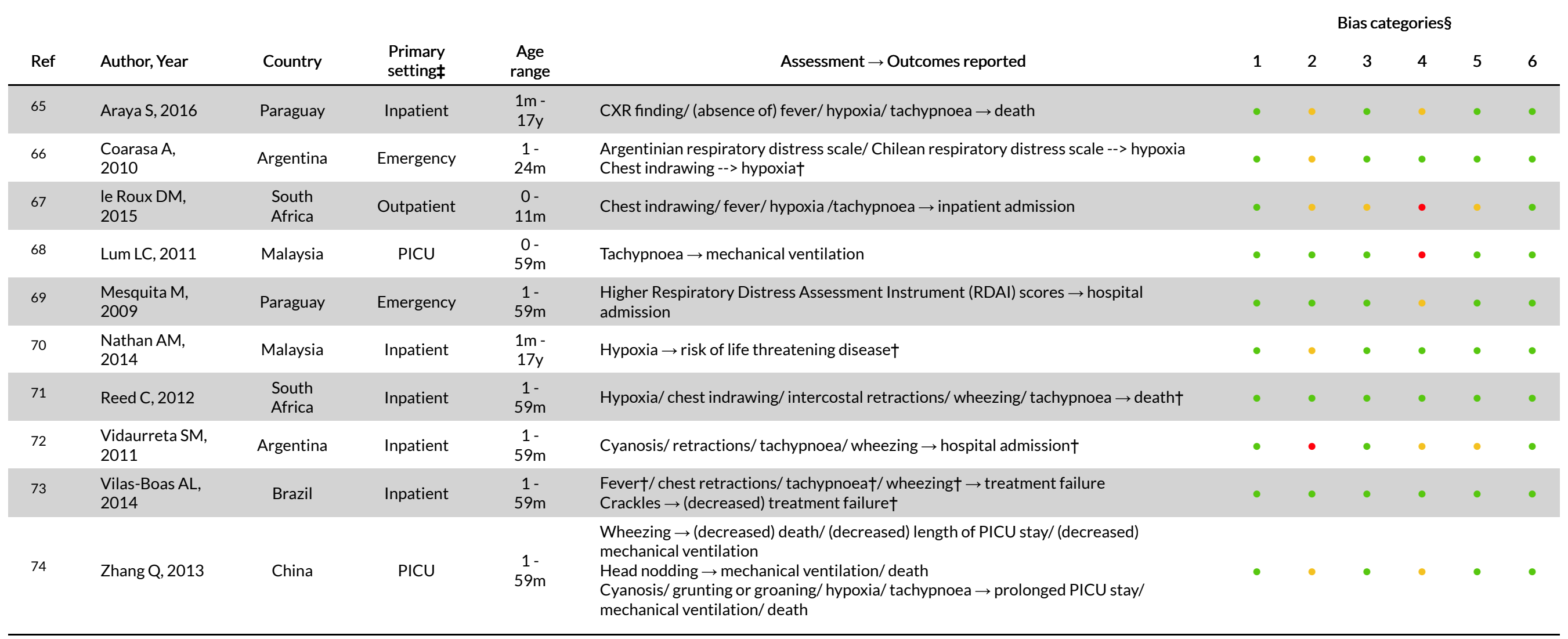

$\bullet=$ low risk of bias, $\ominus=$ unclear risk of bias, $\bullet=$ high risk of bias

$\uparrow$ Association significant on multivariate analysis. $₹$ Primary Setting: Inpatient = Inpatient general ward. PICU = Pediatric Intensive Care Unit. Emergency = Emergency Department. Outpatient = Outpatient clinic.

§ Bias categories: 1.) Comparibility of participants 2.) Confounding variables 3.) Measurement of Exposure 4.) Blinding of outcome assessments 5.) Incomplete outcome data 6.) Selective outcome reporting 
Table 2: Description of studies

\begin{tabular}{|c|c|c|}
\hline Category† & $\begin{array}{l}\text { Count } \\
(n=53)\end{array}$ & (Percent) \\
\hline \multicolumn{3}{|l|}{ Economic Category of Country $\ddagger$} \\
\hline Low Income & 14 & $(26 \%)$ \\
\hline Lower Middle Income & 29 & (55\%) \\
\hline Upper Middle Income & 10 & $(19 \%)$ \\
\hline \multicolumn{3}{|l|}{ Continentł } \\
\hline Africa & 26 & (49\%) \\
\hline Asia & 22 & $(42 \%)$ \\
\hline Europe & 0 & (0\%) \\
\hline North America & 0 & (0\%) \\
\hline South America & 5 & (9\%) \\
\hline \multicolumn{3}{|l|}{ Age of patients (may include more than one category) } \\
\hline Premature infants ( $<37$ weeks gestation) & 3 & (6\%) \\
\hline Neonates ( 0 - 28 days) & 16 & (30\%) \\
\hline Infants (29 days $-<1$ year) & 50 & (94\%) \\
\hline $1-<5$ years & 47 & (89\%) \\
\hline $5-<18$ years & 8 & $(15 \%)$ \\
\hline \multicolumn{3}{|l|}{ Primary Clinical Setting } \\
\hline Outpatient & 7 & $(13 \%)$ \\
\hline Emergency Department & 5 & (9\%) \\
\hline Inpatient General Ward & 35 & (66\%) \\
\hline Pediatric Intensive Care Unit & 3 & (6\%) \\
\hline Neonatal Intensive Care Unit & 3 & (6\%) \\
\hline \multicolumn{3}{|l|}{ Type of Study } \\
\hline Prospective (Cohort, case control, cross sectional) & 37 & (70\%) \\
\hline Retrospective Cohort & 10 & (19\%) \\
\hline Randomized Control Trial & 6 & $(11 \%)$ \\
\hline \multicolumn{3}{|l|}{ Type of Analysis } \\
\hline Univariate & 48 & (91\%) \\
\hline Multivariate & 31 & (58\%) \\
\hline
\end{tabular}

Multiple publications describing the same study ${ }^{22,23}$ as well as these ${ }^{19-21}$ were counted only once. ${ }^{\dagger}$ Most common category reported when $>1$ country in study. $¥$ World Bank. ${ }^{15}$

General characteristics of unique study populations are described in Table 2. Fifty-five percent of studies occurred in lower middle-income countries, and inpatient general wards was the most common clinical settings. Most studies included infants and/or children 1-4 years, with median patient age of 11 months (when reported). The number of study subjects aged less than 5 years ranged from 65 to 16,162 , with a median of 420 (when reported). Bias distribution is shown in Figure 2. The confounding variables domain had the highest risk of bias, with $11 \%$ of publications having "high risk" and 39\% having "unclear risk".

Respiratory assessments are reported in Table 3. Some trends by country income existed, for example, chest indrawing was most frequent in upper middle-income countries while low-income countries were most likely to report grunting and nasal flaring. Hypoxia, defined by most studies as oxygen saturation $<90 \%$, was the most common respiratory assessment and reported equally frequently among all income levels. Twenty publications reported increased mortality in hypoxic vs. non-hypoxic children with ranges of [(3.8\%-29.0\%) vs. (0\%-7.1\%) and odds ratios of (1.6-14.8)]. Nine publications reported prolonged admission or treatment failure in children with hypoxia.

Tachypnea was reported by twenty-six publications (Table 3). Tachypnea was defined by age-appropriate cutoffs in most publications but also as "fast breathing" 59 and "abnormal respiratory rate" 62 which included high and low rates. Upper middle-income country publications reported associations with tachypnea more often than other income categories and country income was associated with the outcome linked to tachypnea. All seven studies reporting an association between tachypnea and hypoxia were conducted 
Table 3. Assessments of respiratory distress reported with statistically significant relationship to clinical outcome

\begin{tabular}{|c|c|c|c|c|c|c|c|c|}
\hline \multirow[b]{2}{*}{ Assessment } & \multicolumn{2}{|c|}{$\begin{array}{l}\text { All Publications } \\
\qquad(n=56)\end{array}$} & \multicolumn{2}{|c|}{$\begin{array}{l}\text { Low Income } \\
\qquad(\mathrm{n}=16)\end{array}$} & \multicolumn{2}{|c|}{$\begin{array}{l}\text { Lower Middle } \\
\text { Income } \\
\quad(n=30)\end{array}$} & \multicolumn{2}{|c|}{$\begin{array}{l}\text { Upper Middle } \\
\text { income } \\
(n=10)\end{array}$} \\
\hline & Count & (\%) & Count & (\%) & Count & (\%) & Count & (\%) \\
\hline \multicolumn{9}{|l|}{ Physical Exam Findings } \\
\hline Chest Indrawing/ Retractions & 21 & $(38 \%)$ & 4 & $(25 \%)$ & 12 & $(40 \%)$ & 5 & $(50 \%)$ \\
\hline Grunting & 11 & $(20 \%)$ & 5 & (31\%) & 5 & $(17 \%)$ & 1 & $(10 \%)$ \\
\hline Cyanosis & 10 & $(18 \%)$ & 3 & $(19 \%)$ & 5 & $(17 \%)$ & 2 & $(20 \%)$ \\
\hline Nasal Flaring & 9 & $(16 \%)$ & 4 & $(25 \%)$ & 5 & $(17 \%)$ & 0 & (0\%) \\
\hline Wheezing & 9 & $(16 \%)$ & 2 & $(13 \%)$ & 3 & $(10 \%)$ & 4 & $(40 \%)$ \\
\hline Head Bobbing & 3 & (5\%) & 2 & $(13 \%)$ & 0 & (0\%) & 1 & $(10 \%)$ \\
\hline $\begin{array}{l}\text { "Respiratory Distress"/ } \\
\text { "Difficulty Breathing" }\end{array}$ & 3 & $(5 \%)$ & 0 & $(0 \%)$ & 3 & $(10 \%)$ & 0 & (0\%) \\
\hline Crepitations, Rales, Crackles & 5 & (9\%) & 4 & $(25 \%)$ & 0 & $(0 \%)$ & 1 & $(10 \%)$ \\
\hline Apnea & 2 & $(4 \%)$ & 0 & (0\%) & 2 & $(7 \%)$ & 0 & (0\%) \\
\hline Other Exam Findings $†$ & 4 & $(7 \%)$ & 1 & (6\%) & 3 & $(10 \%)$ & 0 & (0\%) \\
\hline \multicolumn{9}{|l|}{ Vital Signs } \\
\hline Hypoxia/Low Oxygen Saturation & 28 & $(50 \%)$ & 8 & $(50 \%)$ & 15 & $(50 \%)$ & 5 & $(50 \%)$ \\
\hline $\begin{array}{l}\text { Tachypnoea /Abnormal } \\
\text { Respiratory Rate }\end{array}$ & 26 & $(46 \%)$ & 4 & $(25 \%)$ & 14 & $(47 \%)$ & 8 & (80\%) \\
\hline Fever & 9 & $(16 \%)$ & 2 & $(13 \%)$ & 4 & $(13 \%)$ & 3 & (30\%) \\
\hline \multicolumn{9}{|l|}{ Radiology } \\
\hline Chest Xray Finding & 8 & $(14 \%)$ & 2 & $(13 \%)$ & 5 & $(17 \%)$ & 1 & $(10 \%)$ \\
\hline \multicolumn{9}{|l|}{ Scores } \\
\hline Respiratory Scores & 4 & (7\%) & 1 & $\begin{array}{r}\ddagger \\
(6 \%)\end{array}$ & 0 & (0\%) & 3 & $\begin{array}{r}\S \\
(30 \%)\end{array}$ \\
\hline
\end{tabular}

Publications may have included more than one of each category.

tOther exam findings: Thoracoabdominal breathing, abnormal percussion note, bronchial breath sounds, (lack of) rasping.

‡ Downe’s Score. §Respiratory Distress Assessment Index (Lowell 1987), Argentinean Respiratory Distress Scale and Chilean Respiratory Distress Scale.

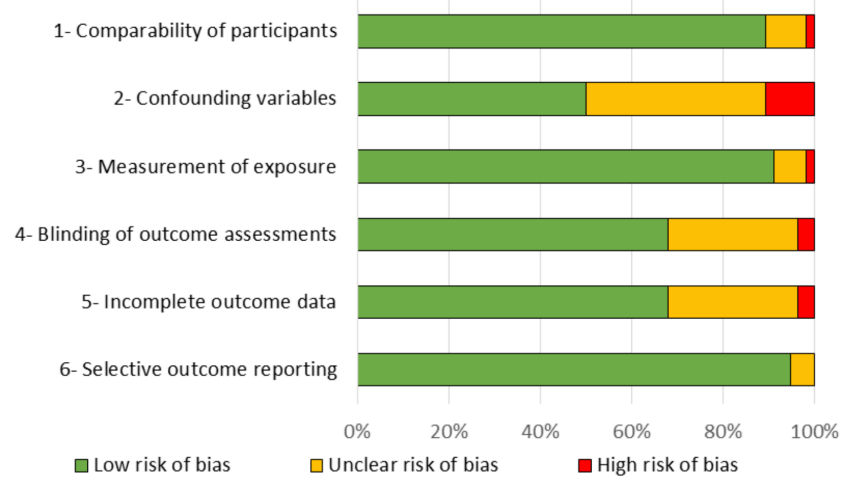

Figure 2: Distribution of types of bias assessments

\footnotetext{
* As defined by Kim et al. ${ }^{18}$
}

in low- or low middle-income countries. ${ }^{29,33,38,55,59,61,75}$ Meanwhile the seven studies reporting increased mortality with tachypnea were conducted in low middle- or upper middle-income countries. ${ }^{42,51,54,64,65,71,74}$ The mortality ranged from $1.7 \%-10.0 \%$ in tachypnoeic children vs.
0.7\%-4.1\% in non-tachypnoeic children. Two other publications instead reported decreased mortality in tachypnoeic children. Naheed (2009) evaluated 4155 Bangladeshi children 2- 59 months hospitalized with pneumonia and found that $95 \%$ of survivors, and $83 \%$ of non-survivors were 
Table 4. Poor clinical outcomes reported with statistically significant relationship to respiratory assessment

\begin{tabular}{|c|c|c|c|c|c|c|c|c|}
\hline \multirow[b]{2}{*}{ Outcome } & \multicolumn{2}{|c|}{$\begin{array}{l}\text { All Publications } \\
\qquad(n=56)\end{array}$} & \multicolumn{2}{|c|}{$\begin{array}{l}\text { Low Income } \\
\qquad(n=16)\end{array}$} & \multicolumn{2}{|c|}{$\begin{array}{l}\text { Lower Middle } \\
\text { Income } \\
(n=30)\end{array}$} & \multicolumn{2}{|c|}{$\begin{array}{l}\text { Upper Middle } \\
\text { Income } \\
(n=10)\end{array}$} \\
\hline & Count & (\%) & Count & (\%) & Count & $(\%)$ & Count & (\%) \\
\hline \multicolumn{9}{|l|}{ Clinical Course } \\
\hline Death & 30 & $(54 \%)$ & 9 & $(56 \%)$ & 17 & $(57 \%)$ & 4 & $(40 \%)$ \\
\hline General Ward Admission & 4 & $(7 \%)$ & 0 & $(0 \%)$ & 1 & $(3 \%)$ & 3 & $(30 \%)$ \\
\hline ICU Admission & 3 & $(5 \%)$ & 0 & $(0 \%)$ & 2 & $(7 \%)$ & 1 & $(10 \%)$ \\
\hline Referral to Higher Level of Care & 1 & $(2 \%)$ & 1 & $(6 \%)$ & 0 & $(0 \%)$ & 0 & $(0 \%)$ \\
\hline Poor Prognosis & 1 & $(2 \%)$ & 0 & $(0 \%)$ & 1 & (3\%) & 0 & $(0 \%)$ \\
\hline \multicolumn{9}{|l|}{ Treatment Course } \\
\hline Length of Hospitalization & 9 & $(16 \%)$ & 3 & (19\%) & 5 & $(17 \%)$ & 1 & $(10 \%)$ \\
\hline Treatment Failure & 7 & $(13 \%)$ & 1 & (6\%) & 5 & $(17 \%)$ & 1 & $(10 \%)$ \\
\hline Prolonged Recovery & 3 & $(5 \%)$ & 2 & $(13 \%)$ & 1 & (3\%) & 0 & $(0 \%)$ \\
\hline \multicolumn{9}{|l|}{ Treatment Requirement } \\
\hline Hypoxia & 13 & $(23 \%)$ & 4 & $(25 \%)$ & 8 & $(27 \%)$ & 1 & $(10 \%)$ \\
\hline Mechanical Ventilation & 3 & $(5 \%)$ & 1 & $(6 \%)$ & 0 & $(0 \%)$ & 2 & $(20 \%)$ \\
\hline $\begin{array}{l}\text { Continous Positive Airway } \\
\text { Pressure }\end{array}$ & 1 & $(2 \%)$ & & (0\%) & 0 & $(0 \%)$ & 1 & $(10 \%)$ \\
\hline $\begin{array}{l}\text { Respiratory Support at } 72 \\
\text { hours }\end{array}$ & 1 & $(2 \%)$ & 1 & (6\%) & 0 & (0\%) & 0 & (0\%) \\
\hline
\end{tabular}

Publications may have included more than one of each category.

tachypnoeic $(P<0.001) .58$ Non-survivors were more likely to have severe pneumonia, but analyses were not adjusted for severity of illness. Emukule (2014) evaluated 3581 Kenyan children less than 5 years hospitalized with respiratory illness. Sixty-four percent of survivors had tachypnea versus $56 \%$ of non-survivors (odds ratio, OR= $0.7,95 \%$ confidence interval, $\mathrm{CI}=0.5-0.9$ ); however, this association was not significant in multivariate analysis. ${ }^{50}$

Clinical outcomes are reported in Table 4. The most commonly reported clinical outcome was death (54\%), followed by hypoxia (23\%), length of hospitalization (16\%) and treatment failure (13\%). Hypoxia was the most commonly reported risk factor associated with mortality (77\%), followed by chest indrawing/retractions, (27\%) and tachypnea (23\%). No trends by income level existed for death, length of hospitalization nor treatment failure. Upper middle-income countries were more likely to report outcomes of general ward admission or need for mechanical ventilation.

Three authors reported risk factors for hypoxia and increased mortality in hypoxemic patients in the same study population. Bassat (2016) evaluated 825 children less than five years of age hospitalized with respiratory distress in Mozambique and found that tachypnea, cyanosis and thoracoabdominal breathing were independently associated with hypoxia, and these hypoxemic children had increased mortality (20\% vs. $7 \%$ ). ${ }^{29}$ Kuti used a population of 420 Gambian children to report risk factors for hypoxia, including head nodding ( $35 \%$ vs $16 \%$ ), grunting ( $61 \%$ vs $23 \%$ ), and cyanosis $(20 \% \text { vs }<1 \%)^{19}$ and increased mortality in hypoxic vs. non hypoxic children (40\% vs $18 \%) .{ }^{20}$ Benet (2017)'s multicenter study of 405 hospitalized children (2 - 60 months old) reported risk factors for hypoxemia including: lower chest indrawing (90\% vs. $77 \%$ ), cyanosis (14\% vs. $5 \%$ ), lack of "rasping” (17\% vs 6\%), and generalized opacification on chest X-ray (CXR) (44\% vs. $28 \%$ ) as well as higher mortality in hypoxemic children ( $9 \%$ vs $2 \%$ ). ${ }^{46}$

\section{DISCUSSION}

To our knowledge, this is the first systematic review of markers of pediatric respiratory distress associated with poor clinical outcomes in LMICs. Our findings underscore substantial heterogeneity in the literature related to this topic and this precluded meta-analysis.

This study demonstrates the following key findings: i) hypoxia, chest indrawing/retractions, and tachypnea are the most commonly reported risk factors for child mortality from respiratory disease in RLS; ii) studies in RLS most frequently report death as the primary outcome of pediatric respiratory illness; iii) hypoxia is a key respiratory assessment and outcome of interest in RLS; and iv) few trends exist related to reporting of respiratory assessments or outcomes based upon country income level.

The results of this review serve several purposes for clinicians and researchers caring for children in RLS: With increasing availability of advanced respiratory support (HFNC and CPAP), clinicians need validated criteria for identifying children likely to benefit from this support and/or in need of transfer to higher levels of care. ${ }^{7}$ It can also help re- 
searchers to develop and implement respiratory scores, choosing predictors based upon available resources and patient population.

Hypoxia, chest indrawing/retractions, and tachypnea were the assessments most commonly associated with mortality in this review. This is a reflection likely of a true physiologic association as well as the frequency with which these assessments are performed and studied in these populations. These assessments are in line with World Health Organization guidelines using tachypnea and chest indrawing as indications for antibiotic therapy and for categorization of pneumonia severity. ${ }^{7}$ A recent meta-analysis from predominantly LMICs found that the strongest predictors of a pneumonia diagnosis in children under 5 years were tachypnea (likelihood ratio, $L R=1.9$ ), chest indrawing $(\mathrm{LR}=1.8)$ as well as grunting $(\mathrm{LR}=1.8)$ and nasal flaring $(\mathrm{LR}=1.8) .{ }^{76}$ While evidence-based diagnosis is important for medication treatment including antibiotics and bronchodilators, determining severity of disease is more essential to guide respiratory treatments and transfer decisions. The heterogeneity of poor clinical outcomes in this review has limited the ability to do a meta-analysis, however some systematic reviews have reported associations with specific clinical outcomes. A recent systematic review in infants less than 60 days in RLS found that the strongest predictors of hospital admission were tachypnea $(\mathrm{OR}=1.5-3.1)$, grunting $(\mathrm{OR}=1.5-2.9)$, severe chest indrawing $(\mathrm{OR}=1.5-8.9)$, and cyanosis $(\mathrm{OR}=1.5-25.8) .{ }^{77}$ Several of these same exam findings have been previously reported to increase the likelihood of hypoxia: cyanosis $(L R=10.4)$, grunting $(L R=2.6)$, nasal flaring ( $\mathrm{LR}=2.2$ ) and chest retraction $(\mathrm{LR}=2.6)$; however authors concluded that "neither single nor combined symptoms and signs have satisfactory performance in predicting hypoxia among young children with acute respiratory infection". ${ }^{10}$ Although our findings emphasize the importance of these common respiratory assessments, the heterogeneity in studies makes identifying optimal predictors for variably resourced clinical settings challenging.

Death was the most commonly reported outcome for respiratory disease in this review. Given that over 800,000 children under five died from lower respiratory diseases in 2017, it is not surprising that reducing mortality remains the focus. $^{2}$ Ongoing inequities in access to respiratory care, such as oxygen and CPAP, contribute to this discrepancy in mortality. ${ }^{78-80}$ We did not find a trend toward reporting nonmortality outcomes as income level increased, in contrast to high-income countries that have transitioned to reporting predominantly non-mortality outcomes. ${ }^{81-83}$ This could reflect high mortality rates in upper middle income countries, making death still the most relevant outcome. Alternatively, it may reflect differences in facility-level resources and mortality risk independent of country income level, given known differences in within-country resource availability and access to care. As more regional estimates of disease burden are reported through the local burden of disease project, we may gain understanding of how within country allocation of resources influences outcomes and potential respiratory assessments. 84,85

The heterogeneity of reported risk factors and outcomes in this review is not unique to markers of respiratory distress or to RLS. Inconsistencies in measurement and report- ing of outcomes are widespread and make it challenging to draw conclusions from many systematic reviews. Many researchers advocate for the development of core outcome sets, which contain a standardized collection of outcomes that should be measured and reported for a particular disease entity. ${ }^{86}$ Development of a core outcome set for pediatric respiratory disease in RLS would need to involve stakeholders across the resource spectrum to ensure that the agreed upon collection of outcomes are relevant to their settings, similar to approaches used by inFACT, a network of investigator - led clinical research consortia that aims to promote international collaboration in critical care research and address barriers in undertaking trials. ${ }^{87}$ A core outcome set could reduce study heterogeneity and improve the ability to use existing data to answer important clinical questions.

This review has a number of limitations. By focusing on publications reporting statistically significant associations with clinical outcomes, we introduced selection bias in addition to existing publication bias favouring significant associations. We minimized this bias by including studies reporting only univariate associations although likely many univariate associations would be not remain significant after multivariate analysis. We used LMICs as a proxy for RLS and categorized studies by country income, which may not equate to comparable resource availability in individual study sites. There was inadequate information about study location (urban vs. rural, national vs. district hospital) or available resources to develop a better stratification schema. We excluded multiple assessments of respiratory illness a priori, including more expensive laboratory or diagnostic tests (PCRs, PFTs), elements of patient history (ie cough) and non-specific danger signs (inability to drink, persistent vomiting, convulsions, lethargy, severe malnutrition) to focus on items most pertinent to respiratory disease in RLS. Only three publications evaluating composite scores were included in this review, which may relate to our search strategy or a paucity of validation of scores in RLS. We excluded specific diagnoses, such as pneumonia or asthma, as valid outcomes because these can be subjective or based on diagnostic criteria that include our assessments of interest (ex. tachypnea for pneumonia). Given the broad nature of the research question, our search strategy identified a large number of abstracts that could only be screened by one reviewer, increasing selection bias. For two publications we were unable to determine if they referred to the same study population, which could affect data in Table 2.

\section{CONCLUSIONS}

In conclusion, this review emphasizes the importance of hypoxia, chest retractions, and tachypnea as risk factors for mortality while highlighting death as the primary outcome of interest for pediatric respiratory disease in RLS. Given wide variability in clinical settings in LMIC, we were unable to determine consistent, validated markers of pediatric respiratory distress that correlate to poor clinical outcomes. Clinicians and researchers throughout LMIC would benefit from development of a core outcomes set to standardize methodology in future studies. More uniform measurement 
of outcomes across RLS should allow for more thorough review with meta-analysis to be performed. An analysis like this has the potential to aid clinicians and researchers in identifying which markers of respiratory distress best identify children at highest risk of clinical deterioration and mortality.

Acknowledgements: Susan Groshong, MLIS; Rachel Umoren, MD MS (collaborator); Krystle Perez, MD MPH (collaborator); Maneesh Batra, MD MPH; and Funding for REDCap Server provided by Institute of Translational Health Science (ITHS) grant support (UL1 TR002319 from NCATS/NIH).

\section{Funding: None.}

Authorship contributions: $\mathrm{AH}, \mathrm{AV}, \mathrm{KP}, \mathrm{KN}$ and RU designed the study. SG carried out the database search. AH, AV, KN, $\mathrm{KP}$ and RU (screened abstracts). AH, AV, KG and KN assessed and extracted data from full text articles. AH prepared tables and figures. AH, AV, KG and $\mathrm{KN}$ wrote and edited the manuscript.

Competing interests: Author $\mathrm{AH}$ is an author of two abstracts in the search. She did not review these abstracts for inclusion. The authors completed the Unified Competing Interest form at www.icmje.org/coi disclosure.pdf (available upon request from the corresponding author), and declare no other conflicts of interest.

\section{Correspondence to:}

Dr. Anna Hedstrom

4800 Sand Point Way NE

Seattle Children's Hospital, FA.2.113

Seattle WA 98105

hedstrom@uw.edu

https://orcid.org/0000-0002-3269-1892 


\section{REFERENCES}

1. Liu L, Oza S, Hogan D, Perin J, Rudan I, Lawn JE, et al. Global, regional, and national causes of child mortality in 2000-13, with projections to inform post-2015 priorities: An updated systematic analysis. Lancet. 2015;385(9966):430-440.

\section{Global Burden of Disease Compare Data} Visualization [Internet]. IHME, University of Washington; 2017. http://vizhub.healthdata.org/gbdcompare.

3. Global Burden of Disease Causes Death Collaborators. Global, regional, and national age-sexspecific mortality for 282 causes of death in; 195 countries and territories, 1980-2017: a systematic analysis for the Global Burden of Disease Study 2017 (vol 392, pg 1736, 2018). Lancet.

2018;392(10160):2170-.

4. Global Burden of Disease Lower Respiratory Infections Collaborators. Estimates of the global, regional, and national morbidity, mortality, and aetiologies of lower respiratory infections in 195 countries, 1990-2016: A systematic analysis for the Global Burden of Disease Study 2016. Lancet Infect Dis. 2018;18(11):1191-1210.

5. Duke T, Cheema B. Paediatric emergency and acute care in resource poor settings. J Paediatr Child Health. 2016;52(2):221-226.

6. World Health Organization, ed. Handbook: Integrated Management of Childhood Illness. Geneva, Switzerland: World Health Organization; 2005.

\section{World Health Organization. Revised WHO} Classification and Treatment of Childhood Pneumonia at Health Facilities: Evidence Summaries. Switzerland; 2014.

8. Justicia-Grande AJ, Pardo Seco J, Rivero Calle I, Martinon-Torres F. Clinical respiratory scales: Which one should we use? Expert Rev Respir Med. 2017;11(12):925-943.

9. Thukral A, Sankar MJ, Chandrasekaran A, Agarwal R, Paul VK. Efficacy and safety of CPAP in low- and middle-income countries. Journal of perinatology: official journal of the California Perinatal Association. 2016;36 Suppl 1:S21-28.

10. Zhang L, Mendoza-Sassi R, Santos JC, Lau J. Accuracy of symptoms and signs in predicting hypoxaemia among young children with acute respiratory infection: A meta-analysis. Int J Tuberc Lung Dis. 2011;15(3):317-325.
11. Rojas-Reyes MX, Granados Rugeles C, CharryAnzola LP. Oxygen therapy for lower respiratory tract infections in children between 3 months and 15 years of age. Cochrane Database Syst Rev. 2014;(12):CD005975. doi:10.1002/14651858.cd00597 5.pub3

12. Bekhof J, Reimink R, Brand PL. Systematic review: Insufficient validation of clinical scores for the assessment of acute dyspnoea in wheezing children. Paediatr Respir Rev. 2014;15(1):98-112.

13. Birken CS, Parkin PC, Macarthur C. Asthma severity scores for preschoolers displayed weaknesses in reliability, validity, and responsiveness. J Clin Epidemiol. 2004;57(11):1177-1181.

14. Moher D, Liberati A, Tetzlaff J, Altman DG, Group P. Preferred reporting items for systematic reviews and meta-analyses: The PRISMA statement. J Clin Epidemiol. 2009;62(10):1006-1012.

15. World Development Indicators [Internet]. The World Bank. 2010. http://data.worldbank.org/data-cat alog/world-development-indicators.

16. Covidence systematic review software [Internet. Veritas Health Innovation. 2019. http://www.covidenc e.org.

17. Harris PA, Taylor R, Thielke R, Payne J, Gonzalez $\mathrm{N}$, Conde JG. Research electronic data capture (REDCap)-a metadata-driven methodology and workflow process for providing translational research informatics support. J Biomed Inform.

2009;42(2):377-381.

18. Kim SY, Park JE, Lee YJ, Seo HJ, Sheen SS, Hahn S, et al. Testing a tool for assessing the risk of bias for nonrandomized studies showed moderate reliability and promising validity. J Clin Epidemiol.

2013;66(4):408-414.

19. Kuti BP, Adegoke SA, Ebruke BE, Howie S, Oyelami OA, Ota M. Determinants of oxygen therapy in childhood pneumonia in a resource-constrained region. ISRN pediatr. 2013;2013:435976.

20. Kuti BP, Adegoke SA, Ebruke BE, Howie S, Oyelami OA, Ota MOC. Risk factors for mortality in childhood pneumonia in a rural West African region. Journal of Pediatric Infectious Diseases. 2013;8(3):131-138. 
21. Kuti BP, Adegoke SA, Oyelami OA, Ota MO. Predictors of prolonged hospitalisation in childhood pneumonia in a rural health centre. SAJCH South African Journal of Child Health. 2014;8(1):11-15.

22. Chisti MJ, Duke T, Robertson CF, Ahmed T, Faruque AS, Ashraf H, et al. Clinical predictors and outcome of hypoxaemia among under-five diarrhoeal children with or without pneumonia in an urban hospital, Dhaka. Bangladesh Trop Med Int Health. 2012;17(1):106-111.

23. Chisti MJ, Duke T, Robertson CF, Ahmed T, Faruque AS, Bardhan PK, et al. Co-morbidity: Exploring the clinical overlap between pneumonia and diarrhoea in a hospital in Dhaka. Bangladesh Ann Trop Paediatr. 2011;31(4):311-319.

24. Chisti MJ, Salam MA, Ashraf H, et al. Clinical risk factors of death from pneumonia in children with severe acute malnutrition in an urban critical care ward of Bangladesh. PLOS ONE. 2013;8(9):e73728. do i:10.1371/journal.pone.0073728

25. Chisti MJ, Salam MA, Ashraf H, et al. Predictors and outcome of hypoxemia in severely malnourished children under five with pneumonia: A case control design. PLOS ONE. 2013;8(1):e51376. doi:10.1371/jou rnal.pone.0051376

26. Acacio S, Verani J, Lanaspa M, et al. Under treatment of pneumonia among children under 5 years of age in a malaria-endemic area: populationbased surveillance study conducted in Manhica district- rural, Mozambique. Int J Infect Dis. 2015;36:39-45.

27. Basnet S, Sharma A, Mathisen M, et al. Predictors of duration and treatment failure of severe pneumonia in hospitalized young Nepalese children. Thorne C, ed. PLoS ONE. 2015;10(3):e0122052. doi:1 0.1371/journal.pone.0122052

28. Bassat Q, Guinovart C, Sigauque B, Aide P, Sacarlal J, Nhampossa T, et al. Malaria in rural Mozambique. Part II: Children admitted to hospital. Malar J. 2008;7:37.

29. Bassat Q, Lanaspa M, Machevo S, O'CallaghanGordo C, Madrid L, Nhampossa T, et al. Hypoxaemia in Mozambican children $<5$ years of age admitted to hospital with clinical severe pneumonia: Clinical features and performance of predictor models. Trop Med Int Health. 2016;21(9):1147-1156.

30. Graham SM, Mankhambo L, Phiri A, Kaunda S, Chikaonda T, Mukaka M, et al. Impact of human immunodeficiency virus infection on the etiology and outcome of severe pneumonia in Malawian children. Pediatr Infect Dis J. 2011;30(1):33-38.
31. John BM, Venkateshwar V, Dagar V. Predictors of outcome in neonates with respiratory distress. Journal of Nepal Paediatric Society. 2015;35(1):31-37.

32. King C, Colbourn T, Mankhambo L, et al. Nontreatment of children with community health workerdiagnosed fast-breathing pneumonia in rural Malawi: Exploratory subanalysis of a prospective cohort study. BMJ Open. 2016;6(11):e011636. doi:10.1136/bmjope $\underline{\mathrm{n}-2016-011636}$

33. McCollum ED, Bjornstad E, Preidis GA, Hosseinipour MC, Lufesi N. Multicenter study of hypoxemia prevalence and quality of oxygen treatment for hospitalized Malawian children. Trans R Soc Trop Med Hyg. 2013;107(5):285-292.

34. Nantanda R, Hildenwall H, Peterson S, KadduMulindwa D, Kalyesubula I, Tumwine JK. Bacterial aetiology and outcome in children with severe pneumonia in Uganda. Ann Trop Paediatr. 2008;28(4):253-260.

35. Nantanda R, Ostergaard MS, Ndeezi G, Tumwine JK. Clinical outcomes of children with acute asthma and pneumonia in Mulago hospital, Uganda: A prospective study. BMC Pediatr. 2014;14:285.

36. Ramakrishna B, Graham SM, Phiri A, Mankhambo L, Duke T. Lactate as a predictor of mortality in Malawian children with WHO-defined pneumonia. Arch Dis Child. 2012;97(4):336-342.

37. Sigauque B, Roca A, Bassat Q, Morais L, Quinto L, Berenguera A, et al. Severe pneumonia in Mozambican young children: Clinical and radiological characteristics and risk factors. J Trop Pediatr. 2009;55(6):379-387.

38. Wandeler G, Pauchard JY, Zangger E, Diawara H, Gehri M. Which clinical signs predict hypoxaemia in young Senegalese children with acute lower respiratory tract disease? Paediatr Int Child Health. 2015;35(1):65-68.

39. Abdulkadir MB, Ibraheem RM, Gobir AA, Johnson WB. Hypoxaemia as a measure of disease severity in young hospitalised Nigerian children with pneumonia: A cross-sectional study. SAJCH South African Journal of Child Health. 2015;9(2):53-56.

40. Addo-Yobo E, Anh DD, El-Sayed HF, Fox LM, Fox MP, MacLeod W, et al. Outpatient treatment of children with severe pneumonia with oral amoxicillin in four countries: The MASS study. Trop Med Int Health. 2011;16(8):995-1006. 
41. Agweyu A, Kibore M, Digolo L, Kosgei C, Maina V, Mugane S, et al. Prevalence and correlates of treatment failure among Kenyan children hospitalised with severe community-acquired pneumonia: A prospective study of the clinical effectiveness of WHO pneumonia case management guidelines. Trop Med Int Health.

2014;19(11):1310-1320.

42. Agweyu A, Lilford RJ, English M. Clinical Information Network Author G. Appropriateness of clinical severity classification of new WHO childhood pneumonia guidance: A multi-hospital, retrospective, cohort study. Lancet Glob Health. 2018;6(1):e74-e83.

43. Asghar R, Banajeh S, Egas J, et al.

Chloramphenicol versus ampicillin plus gentamicin for community acquired very severe pneumonia among children aged 2-59 months in low resource settings. Multicentre randomised controlled trial (SPEAR study) Bmj. 2008;336(7635):80-84.

44. Ashraf H, Alam NH, Chisti MJ, Salam MA, Ahmed $\mathrm{T}$, Gyr N. Observational follow-up study following two cohorts of children with severe pneumonia after discharge from day care clinic/hospital in Dhaka, Bangladesh. BMJ Open. 2012;2(4).

45. Awasthi S, Agarwal G, Kabra SK, et al. Does 3-day course of oral amoxycillin benefit children of nonsevere pneumonia with wheeze: A multicentric randomised controlled trial. Clifford T, ed. PLOS ONE. 2008;3(4):e1991. doi:10.1371/journal.pone.0001991

46. Benét T, Picot VS, Awasthi S, Pandey N, Bavdekar A, Kawade A, et al. Severity of pneumonia in under 5-year-old children from developing countries: A multicenter, prospective, observational study. American Journal of Tropical Medicine and Hygiene. 2017;97(1):68-76.

47. Bharti B, Kaur L, Bharti S. Role of chest X-ray in predicting outcome of acute severe pneumonia. Indian Pediatr. 2008;45(11):893-898.

48. Chudasama RK, Patel UV, Verma PB, Agarwal P, Bhalodiya S, Dholakiya D. Clinical and epidemiological characteristics of 2009 pandemic influenza A in hospitalized pediatric patients of the Saurashtra region, India. World J Pediatr. 2012;8(4):321-327.

49. El Kholy A, Mostafa N, Ali A, El-Sherbini S, Ismail $\mathrm{R}$, Magdy, et al. R. Risk factors of prolonged hospital stay in children with viral severe acute respiratory infections. Journal of Infection in Developing Countries. 2014;8(10):1285-1293.
50. Emukule GO, McMorrow M, Ulloa C, et al. Predicting mortality among hospitalized children with respiratory illness in Western Kenya, 2009-2012. Metcalfe JZ, ed. PLOS ONE. 2014;9(3):e92968. doi:1 0.1371/journal.pone.0092968

51. Gowraiah V, Awasthi S, Kapoor R, Sahana D, Venkatesh P, Gangadhar B, et al. Can we distinguish pneumonia from wheezy diseases in tachypnoeic children under low-resource conditions? A prospective observational study in four Indian hospitals. Arch Dis Child. 2014;99(10):899-906.

52. Hazir T, Nisar YB, Abbasi S, Ashraf YP, Khurshid J, Tariq P, et al. Comparison of oral amoxicillin with placebo for the treatment of world health organization-defined nonsevere pneumonia in children aged 2-59 months: A multicenter, doubleblind, randomized, placebo-controlled trial in pakistan. Clin Infect Dis. 2011;52(3):293-300.

53. Ibraheem RM, Johnson WB, Abdulkarim AA. Hypoxaemia in hospitalised under-five Nigerian children with pneumonia. West Afr J Med. 2014;33(1):37-43.

54. Jroundi I, Mahraoui C, Benmessaoud R, Moraleda C, Tligui H, Seffar M, et al. Risk factors for a poor outcome among children admitted with clinically severe pneumonia to a university hospital in Rabat, Morocco. Int J Infect Dis. 2014;28:164-170.

55. Mahajan V, Tiwari M, Arya A, Tiwari A, Chawla D, Saini SS. Clinical predictors of hospital admission in acute lower respiratory tract infection in 2 months to 2-year-old children. Respirology. 2016;21(2):350-356.

56. Morgan MC, Maina B, Waiyego M, Mutinda C, Aluvaala J, Maina M, et al. Pulse oximetry values of neonates admitted for care and receiving routine oxygen therapy at a resource-limited hospital in Kenya. J Paediatr Child Health. 2017;27:27.

57. Mwaniki MK, Nokes DJ, Ignas J, Munywoki P, Ngama M, Newton CR, et al. Emergency triage assessment for hypoxaemia in neonates and young children in a Kenyan hospital: An observational study. Bull World Health Organ. 2009;87(4):263-270.

58. Naheed A, Saha SK, Breiman RF, Khatun F, Brooks WA, El Arifeen S, et al. Multihospital surveillance of pneumonia burden among children aged $<5$ years hospitalized for pneumonia in Bangladesh. Clin Infect Dis. 2009:82-89.

59. Orimadegun AE, Ogunbosi BO, Carson SS. Prevalence and predictors of hypoxaemia in respiratory and non-respiratory primary diagnoses among emergently ill children at a tertiary hospital in south western Nigeria. Trans R Soc Trop Med Hyg. 2013;107(11):699-705. 
60. Quiambao BP, Ruutu PJ, Ladesma EA, Gozum LS, Inobaya MT, Lupisan SP, et al. Pneumonia among young infants in rural Southeast Asia (Bohol Island, Philippines. Trop Med Int Health.

2009;14(12):1457-1466.

61. Rao YK, Midha T, Kumar P, Tripathi VN, Rai OP. Clinical predictors of hypoxemia in Indian children with acute respiratory tract infection presenting to pediatric emergency department. World J Pediatr. 2012;8(3):247-251.

62. Singla G, Bains HS, Soni RK. Evaluation of clinical parameters for the prediction of hospital stay in neonates admitted in a tertiary referral hospital in a developing country. Iranian Journal of Neonatology. 2015;6(3):6-11.

63. Webb C, Ngama M, Ngatia A, et al. Treatment failure among kenyan children with severe pneumonia-a cohort study. The Pediatric Infectious Disease Journal. 2012;31(9):e152-e157. doi:10.1097/in f.0b013e3182638012

64. Wilson PT, Baiden F, Brooks JC, et al. Continuous positive airway pressure for children with undifferentiated respiratory distress in Ghana: An open-label, cluster, crossover trial. The Lancet Global Health. 2017;5(6):e615-e623. doi:10.1016/s2214-109 x(17)30145-6

65. Araya S, Lovera D, Zarate C, Apodaca S, Acuna J, Sanabria G, et al. Application of a Prognostic Scale to Estimate the Mortality of Children Hospitalized with Community-acquired Pneumonia. Pediatr Infect Dis J. 2016;35(4):369-373.

66. Coarasa A, Giugno H, Cutri A, Loto Y, Torres F, Giubergia V, et al. Validation of a clinical prediction tool to evaluate severity in children with wheezing. Arch Argent Pediatr. 2010;108(2):116-123.

67. le Roux DM, Myer L, Nicol MP, Zar HJ. Incidence and severity of childhood pneumonia in the first year of life in a South African birth cohort: The Drakenstein Child Health Study. The Lancet Global Health. 2015;3(2):e95-e103. doi:10.1016/s2214-109 x(14)70360-2

68. Lum LC, Abdel-Latif ME, de Bruyne JA, Nathan AM, Gan CS. Noninvasive ventilation in a tertiary pediatric intensive care unit in a middle-income country. Pediatr Crit Care Med. 2011;12(1):e7-13.

69. Mesquita M, Castro-Rodríguez JA, Heinichen L, Fariña E, Iramain R. Single oral dose of dexamethasone in outpatients with bronchiolitis: A placebo controlled trial. Allergol Immunopathol (Madr. 2009;37(2):63-67.
70. Nathan AM, Rani F, Lee RJY, et al. Clinical risk factors for life-threatening lower respiratory tract infections in children: A retrospective study in an urban city in Malaysia. Tang JW, ed. PLOS ONE. 2014;9(10):e111162. doi:10.1371/journal.pone.01111 $\underline{62}$

71. Reed C, Madhi SA, Klugman KP, et al. Development of the Respiratory Index of Severity in Children (RISC) score among young children with respiratory infections in South Africa. Jhaveri R, ed. PLoS ONE. 2012;7(1):e27793. doi:10.1371/journal.po ne.0027793

72. Vidaurreta SM, Marcone DN, Ellis A, Ekstrom J, Cukier D, Videla C, et al. Acute viral respiratory infection in children under 5 years: Epidemiological study in two centers in Buenos Aires, Argentina. Arch Argent Pediatr. 2011;109(4):296-304.

73. Vilas-Boas AL, Fontoura MS, Xavier-Souza G, Araujo-Neto CA, Andrade SC, Brim RV, et al. Comparison of oral amoxicillin given thrice or twice daily to children between 2 and 59 months old with non-severe pneumonia: A randomized controlled trial. J Antimicrob Chemother. 2014;69(7):1954-1959.

74. Zhang Q, Guo Z, Bai Z, MacDonald NE. A 4 year prospective study to determine risk factors for severe community acquired pneumonia in children in southern China. Pediatr Pulmonol. 2013;48(4):390-397.

75. Chisti MJ, Salam MA, Ashraf H, Faruque ASG, Bardhan PK, Shahid A, et al. Predictors and Outcome of Hypoxemia in Severely Malnourished Children under Five with Pneumonia: A Case Control Design. PLOS ONE. 2013;8(1).

76. Rambaud-Althaus C, Althaus F, Genton B, D'Acremont V. Clinical features for diagnosis of pneumonia in children younger than 5 years: A systematic review and meta-analysis. Lancet Infect Dis. 2015;15(4):439-450.

77. Opiyo N, English M. What clinical signs best identify severe illness in young infants aged 0-59 days in developing countries? A systematic review. Arch Dis Child. 2011;96(11):1052-1059.

78. Duke T. Pneumonia and bronchiolitis in developing countries. Arch Dis Child. 2014;99(10):892-893.

79. Ginsburg AS, Van Cleve WC, Thompson MI, English M. Oxygen and pulse oximetry in childhood pneumonia: A survey of healthcare providers in resource-limited settings. J Trop Pediatr. 2012;58(5):389-393. 
80. Nabwire J, Namasopo S, Hawkes M. Oxygen Availability and Nursing Capacity for Oxygen Therapy in Ugandan Paediatric Wards. J Trop Pediatr. 2018;64(2):97-103.

81. Thomson J, Ambroggio L, Murtagh Kurowski E, Statile A, Graham C, Courter JD, et al. Hospital outcomes associated with guideline-recommended antibiotic therapy for pediatric pneumonia. J Hosp Med. 2015;10(1):13-18.

82. Yehya N, Thomas NJ. Relevant Outcomes in Pediatric Acute Respiratory Distress Syndrome Studies. Front Pediatr. 2016;4:51.

83. Quasney MW, López-Fernández YM, Santschi M, Watson RS. The outcomes of children with pediatric acute respiratory distress syndrome: proceedings from the Pediatric Acute Lung Injury Consensus Conference . Pediatric Critical Care Medicine. 2015;16:S118-S131. doi:10.1097/pcc.00000000000004 $\underline{38}$
84. Dwyer-Lindgren L. Mapping HIV prevalence in sub-Saharan Africa between 2000 and 2017. Nature. 2019;570:189-193.

85. Weiss DJ, Lucas TCD, Nguyen M, Nandi AK, Bisanzio D, Battle KE, et al. Mapping the global prevalence, incidence, and mortality of Plasmodium falciparum, 2000-17: A spatial and temporal modelling study. Lancet. 2019.

86. Clarke M, Williamson PR. Core outcome sets and systematic reviews. Syst Rev. 2016;5:11.

87. InFACT. Promoting international collaboration 2020. https://www.infactglobal.org/our-vision. Published May 6, 2020. 


\section{SUPPLEMENTARY MATERIALS}

\section{Appendix 1}

Download: https://www.joghr.org/article/14136-markers-of-pediatric-respiratory-distress-predictive-of-pooroutcome-in-low-and-middle-income-countries-a-systematic-review/attachment/39268.docx

\section{Appendix 2}

Download: https://www.joghr.org/article/14136-markers-of-pediatric-respiratory-distress-predictive-of-pooroutcome-in-low-and-middle-income-countries-a-systematic-review/attachment/39269.xlsx 